\title{
Toxina botulínica no tratamento da paralisia facial: um tratamento reabilitador minimamente invasivo
}

\author{
Botulinum toxin in the treatment of facial paralysis: a minimally invasive rehabilitation treatment \\ Toxina botulínica en el tratamiento de la parálisis facial: un tratamiento de rehabilitación
} mínimamente invasivo

Recebido: 19/04/2021 | Revisado: 26/04/2021 | Aceito: 26/04/2021 | Publicado: 11/05/2021

\author{
Juliana Roberta Neves Ênia \\ ORCID: https://orcid.org/0000-0003-1981-1156 \\ Faculdade Patos de Minas, Brasil \\ E-mail: juliana.01386@alunofpm.com.br \\ Jéssica Gonçalves de Almeida Fernandes \\ ORCID: https://orcid.org/0000-0001-6170-1984 \\ Faculdade Patos de Minas, Brasil \\ E-mail: jessica.09041@alunofpm.com.br \\ Fernando Nascimento \\ ORCID: https://orcid.org/0000-0003-3568-3887 \\ Faculdade Patos de Minas, Brasil \\ E-mail: fernando.nascimento@ faculdadepatosdeminas.edu.br \\ Luciana de Araújo Mendes Silva \\ ORCID: https://orcid.org/0000-0003-0311-1323 \\ Faculdade Patos de Minas, Brasil \\ E-mail: luciana.silva@faculdadepatosdeminas.edu.br \\ Tais Reis \\ ORCID: https://orcid.org/0000-0003-3936-7312 \\ Faculdade Patos de Minas, Brasil \\ E-mail: taisareis@yahoo.com \\ Lia Dietrich \\ ORCID: https://orcid.org/0000-0001-7887-8591 \\ Clínica Privada, Brasil \\ E-mail: dietrichlia.ld@gmail.com
}

\begin{abstract}
Resumo
A toxina botulínica é obtida através de uma bactéria anaeróbica conhecida como Clostridium botulinum, e pode ser utilizada para diversos fins, desde estético até o tratamento de diversas doenças, como na reabilitação de pacientes com paralisia facial. O objetivo do presente trabalho é mostrar a importância e os efeitos da aplicação da toxina botulínica em pacientes com paralisia facial, bem como analisar as indicações e aplicabilidades clínicas do produto. Foram realizadas buscas em bases de dados como Google Acadêmico, Lilacs, Scielo, preferencialmente entre o período de 2000 a 2020, buscando as palavras chaves: "Paralisia Facial", "Toxina botulínica", "Simetria da face", em que foram selecionados artigos com textos completos, escritos em português e inglês. $\mathrm{O}$ uso da toxina botulínica na reabilitação de pacientes com paralisia facial vem aumentando cada vez mais e os estudos a respeito do produto estão em crescimento, de forma a se alcançar um protocolo único de utilização, mesmo que ainda pode-se notar escassez desses estudos. A reabilitação dos pacientes com paralisia facial utilizando a toxina botulínica é eficaz e segura, sendo uma opção para melhorar a assimetria facial.
\end{abstract}

Palavras-chave: Assimetria facial; Nervo facial; Paralisia facial; Toxinas botulínicas tipo A.

\begin{abstract}
Botulinum toxin is attained through an anaerobic bacteria known as Clostridium botulinum and can be used for several purposes, from aesthetic purposes to the treatment of various diseases, such as in the rehabilitation of patients with facial paralysis. The aim of the present work is to show the importance and the effects of the application of botulinum toxin in patients with facial paralysis, as well as to analyze the indications and clinical applicability of the product. Researches were carried out from databases such as Google Scholar, Lilacs, Scielo, preferably between the period of 2000 and 2020, by searching for the following keywords: "Facial Paralysis", "Botulinum toxin", "Facial symmetry", in which articles were selected with complete texts, written in Portuguese and in English. The use of botulinum toxin in the rehabilitation of patients with facial paralysis is raising more and more, and studies on the product are growing in order to achieve a single use protocol, even though it's still noticeable the lackness of these
\end{abstract}


studies. The rehabilitation of patients with facial paralysis who use botulinum toxin is effective and safe, and it can be na option to improve facial asymmetry.

Keywords: Facial asymmetry; Facial nerve; Facial paralysis; Botulinum toxin type A.

\section{Resumen}

La toxina botulínica se obtiene a través de una bacteria anaeróbica conocida como Clostridium botulinum, y puede utilizarse para diversos fines, desde la estética hasta el tratamiento de diversas enfermedades, como en la rehabilitación de pacientes con parálisis facial. El objetivo de este trabajo es mostrar la importancia y los efectos de la aplicación de la toxina botulínica en pacientes con parálisis facial, así como analizar las indicaciones y la aplicabilidad clínica del producto. Las búsquedas se realizaron en bases de datos como Google Académico, Lilacs, Scielo, preferentemente entre el período 2000 a 2020, buscando las palabras clave: "Parálisis facial", "Toxina botulínica", "Simetría facial", en las que se seleccionaron artículos con textos completos, escritos en portugués e inglés. El uso de la toxina botulínica en la rehabilitación de pacientes con parálisis facial se ha ido incrementando cada vez más, y los estudios sobre el producto son cada vez mayores, con el fin de lograr un protocolo único de uso, aunque todavía se nota una escasez de estos estudios. La rehabilitación de pacientes con parálisis facial mediante toxina botulínica es eficaz y segura, siendo una opción para mejorar la asimetría facial.

Palabras clave: Asimetría facial; Nervio facial; Parálisis facial; Toxina botulínica tipo A.

\section{Introdução}

A toxina botulínica (TxB) é obtida através de uma bactéria anaeróbica conhecida como Clostridium botulinum, e pode ser utilizada para diversos fins, desde estético até o tratamento de doenças, permitindo melhora na qualidade de vida do indivíduo (Dall'Magro et al., 2015). O fármaco provoca a diminuição da tonicidade dos músculos, de forma temporária, promovendo suavidade nas expressões (Dall'Magro et al., 2015).

$\mathrm{Na}$ Odontologia a toxina tem se tornado o fármaco de escolha no tratamento de diversas disfunções orais e faciais, mas é necessário estar ciente do protocolo de utilização para que o tratamento seja eficaz e executado com segurança (Dall'Magro et al., 2015). A neurotoxina botulínica é um produto altamente eficaz e potente que pode ser usado de forma terapêutica, e dentro da odontologia ela tem sido utilizada em diversos tratamentos, como no equilíbrio facial em pacientes que apresentam paralisia facial (Serrera-Figallo et al., 2020). Com isso os pacientes passam a ter um condicionamento da atividade muscular, promovendo um equilíbrio na simetria da face, através de um tratamento menos invasivo que melhora além de questões funcionais, a auto-estima e questões psicológicas do indivíduo (Serrera-Figallo et al., 2020).

Pode-se notar resultados da utilização da toxina botulínica satisfatórios e seguros em todos os tratamentos realizados com a mesma, porém é necessário conhecimento e treinamento adequado do profissional para a realização dos procedimentos (Brennan \& Hickey, 2019).

Sendo assim, justifica-se a realização deste trabalho de pesquisa, pois a aplicação da toxina botulínica é de grande importância para minimizar os efeitos físicos de assimetria da face de indivíduos com paralisia facial (Marciano, Aguiar, Vieira \& Magalhães, 2014). Em relação ao benefício, permite aos pacientes um equilíbrio e controle das atividades musculares, buscando a simetria da face perdida (Marciano et al., 2014). A toxina botulínica vem ganhando espaço em diversos campos da saúde humana, e seu uso tem sido cada vez maior dentro do campo odontológico, podendo ser utilizada tanto com objetivo estético como terapêutico, sempre tendo em vista a saúde e bem estar do paciente (Marciano et al., 2014).

Por fim, justifica-se a escolha desse tema pela presença de um familiar que possui a paralisia facial e que durante muito tempo não conhecia os benefícios da toxina botulínica, e hoje, faz a aplicação da mesma para melhoria da simetria da face, onde consequentemente, devolveu ao mesmo a segurança no convívio social.

Sendo assim, a realização deste trabalho de pesquisa busca mostrar que a aplicação da toxina botulínica é de grande importância para minimizar o desequilíbrio na feição de pessoas que apresentam paralisia facial, dando ao indivíduo mais segurança para o convívio social, tento em vista os benefícios oferecidos por ela. Bem como analisar a indicação e aplicabilidade da toxina botulínica no tratamento de pacientes com paralisia facial. 


\section{Metodologia}

Este estudo foi realizado por meio da revisão narrativa da literatura e apresenta caráter qualitativo, em que é importante adequada interpretação do pesquisador, levando em consideração suas opiniões sobre o assunto estudado (Pereira et al., 2018). O trabalho foi realizado através da análise de artigos científicos, livros, monografias, revistas, dissertações e teses, sendo que o acesso a estes conteúdos serão possíveis através de livros presentes na biblioteca da Faculdade Patos de Minas e sites da busca, como o Google Acadêmico, Scielo, Lilacs, Pubmed, entre outros.

Foi utilizado para busca palavras-chaves como: paralisia facial, toxina botulínica, simetria da face, limitações funcionais, implicações sociais, botox, tratamento minimamente invasivo e keywords: facialparalysis, symmetryofthe face, socialimplications. Os materiais que foram utilizados, estão, preferencialmente, no período de 2000 a 2020 , e encontram-se na língua portuguesa e inglesa.

\section{Revisão da literatura}

\subsection{Ação da toxina botulínica nos tecidos}

A toxina botulínica, também conhecida como Botox e BoNT, foi descoberta em 1895 por Van Ermengem, um bacteriologista que descobriu a substância neurotóxica mais importante e intensa já vista, na qual a bactéria tem a capacidade de produzir uma proteína capaz de gerar a neurotoxina que pode causar o relaxamento da musculatura nos locais onde é aplicada (Carruthers, 2003).

Anos após a descoberta da toxina, em 1950, Brooks divulgou que ela tem a capacidade de promover o relaxamento dos músculos através do bloqueio na liberação de acetilcolina que ocorre nos neurônios do tipo motores (Carruthers, 2003). E apenas em 1980 surgiu o primeiro trabalho publicado sobre o uso da toxina botulínica em humanos com finalidade terapêutica (Carruthers, 2003).

A Clostridium botulinum, é frequentemente encontrada em ambientes marinhos e no solo em qualquer parte do mundo, e trata-se de uma bactéria do tipo anaeróbica Gram-positiva que apresenta formato de esporo, sendo ela responsável pela produção de uma das mais potentes e poderosas toxinas originadas através da fermentação bacteriana, a toxina botulínica (Colhado, Boeing, \& Ortega, 2009).

Através da fermentação da $C$. botulinum foram encontrados oito sorotipos que são diferentes imunologicamente, sendo que eles variam entre si no que diz respeito à potência de cada um deles, o mecanismo de ação e as proteínas encontradas no interior da célula (Colhado et al., 2009). Mas, independente do sorotipo todos promovem o bloqueio da acetilcolina liberada nas terminações nervosas (Colhado et al., 2009). O sorotipo A é o mais potente de todos eles e o mais estudado com finalidade terapêutica, e por isso apresenta maior quantidade de estudos a seu respeito, sendo ela a primeira a ser desenvolvida para ser usada clinicamente (Colhado et al., 2009; Domingos, 2006).

Sem promover alteração da condução neural ou a síntese e o armazenamento de acetilcolina, o sorotipo A, também chamado de toxina botulínica do tipo A, é uma neurotoxina que promove o bloqueio da liberação de acetilcolina que ocorre nos terminais nervosos de sinapses colinérgicas (Unno, Sakata, \& Issy, 2005).

A ação da toxina botulínica (TxB), ou seja, a inibição e bloqueio da acetilcolina, se dá em diversas etapas, sendo que na primeira delas ela se une de forma irreversível aos receptores da membrana pré-sináptica do terminal nervoso motor (Unno et al., 2005). Para determinar uma contração dos músculos de maneira seletiva, logo após a primeira etapa acontece então o processo de endocitose, o que fará com que no citoplasma dos neurônios ocorra a liberação de parte da molécula, e por fim, acontece de forma funcional a desnervação dos músculos (Unno et al., 2005). Quando a aplicação da toxina botulínica é realizada de forma correta, nos locais certos e em doses adequadas na musculatura, ela provocará parcialmente a desnervação e diminuirá a contração muscular sem gerar paralisia definitiva e completa (Unno et al., 2005). 
Para que o efeito da TxB ocorra adequadamente o processo deve passar por algumas fases, sendo elas a ligação, a internalização, traslocação da membrana e a atividade de proteases, sendo que a toxina precisa entrar com total integridade na terminação nervosa para exercer o seu efeito e função (Domingos, 2006). A ligação que será realizada pela TxB é exclusiva para terminais nervosos colinérgicos e trata-se de uma ligação irreversível, que será interceptada pela cadeira pesada, sendo que a cadeira leve é responsável por promover a toxicidade dentro da célula (Domingos, 2006).

O tempo de ação da toxina botulínica nos tecidos varia bastante e pode começar em alguns dias e demorar até 2 semanas para fazer o seu efeito, e o tempo de duração dos efeitos podem ser de 6 semanas podendo chegar a até 6 meses, sendo estabelecido uma média de 3 a 4 meses de duração da ação da toxina (Unno et al., 2005). Em exames histológicos podese observar que durante o período em que a toxina apresenta o seu efeito mais intensificado, ocorre atrofia da musculatura e modificação das fibras musculares (Unno et al., 2005). Ao final da ação e dos efeitos da toxina, surge o período de recuperação em que ocorrerá novamente a inervação, acontecerá a formação de brotos e de novas placas terminais de tamanho menor, o que permitirá reverter a paralisia gerada localmente (Unno et al., 2005).

Cada músculo apresenta a dose recomendada para que a toxina seja aplicada de forma apropriada, e caso ocorra a aplicação de uma dose maior do que a padronizada, o risco principal é de que ocorra fraqueza muscular (Unno et al., 2005). Além de fraqueza no músculo em que ocorreu a aplicação de doses além das recomendadas, também pode ocorrer o mesmo nos músculos próximos, porém é raro ocorrer complicações quando as doses são aplicadas nas localizações corretas e em quantidades adequadas (Unno et al., 2005).

Existe uma relação entre a dose de toxina botulínica que será aplicada e o tempo de duração dos efeitos que serão produzidos, porém apenas quando quantidades relativamente baixas são aplicadas que essa correlação existe (Dressler, Saberi, \& Barbosa, 2005). A ação da TxB parece saturar cerca de três meses após a aplicação quando quantidades mais altas são administradas (Dressler et al., 2005).

Quando a aplicação da toxina botulínica é feita em um músculo que apresenta grande atividade, ocorrerá uma diminuição no tamanho do músculo em que a toxina foi injetada pela paresia que será induzida (Dressler et al., 2005). Em caso de hipertrofia muscular causada pela grande atividade durante um longo período de tempo, a aplicação da toxina botulínica pode voltar o músculo ao seu tamanho normal através da paresia que será provocada na musculatura (Dressler et al., 2005). Quando a administração e injeção da toxina botulínica é feita por um longo período de tempo, a musculatura pode apresentar atrofia muscular real, porém essa consequência não trata-se de um dos efeitos obrigatórios que a administração da toxina devem trazer, e por isso não é um ponto a ser levado em consideração para determinar a eficácia do produto (Dressler et al., 2005).

Quando a toxina botulínica é aplicada sobre o tecido muscular, o primeiro efeito gerado sobre a musculatura ocorre na função do neurônio motor alfa, que faz com que as fibras presentes nos músculos sejam estimuladas (Dressler et al., 2005; Freund \& Schwartz, 2003). Entretanto, pode agir também sobre os neurônios motores do tipo gama, que são responsáveis pela inervação do fuso da musculatura, e sua inibição provoca a diminuição do tônus muscular por causa da redução à reação sobre o neurônio motor alfa, gerado pelo fuso muscular (Dressler et al., 2005; Freund \& Schwartz, 2003). Com a aplicação da TxB o efeito clínico mais observado é fraqueza e paralisia muscular dependendo da dose administrada na musculatura, o que ocorrerá através da ação nos neurônios motores (Dressler et al., 2005; Freund \& Schwartz, 2003).

A administração da TxB disponível para uso no mercado, quando aplicada em pequeno intervalo de tempo, em doses altas e com grande carga protéica, podem levar e aumentam as chances do surgimento de anticorpos neutralizantes (Mejia, Vuong, \& Jankovic, 2005). Foi observado no tratamento de distonias o desenvolvimento desses anticorpos (neutralizantes) que funcionam contra a TxB, que surgiram após o início do tratamento por volta do primeiro ao quarto ano (Mejia et al., 2005). A prevalência de ocorrência do desenvolvimento dos anticorpos é bem parecida tanta em adultos como em crianças (Mejia et al., 
2005). A consequência da formação dos anticorpos neutralizantes é que eles provocam a falha terapêtica parcial, em que o tempo de ação e a permanência dos efeitos terapêuticos produzidos são diminuídos depois de poucas aplicações (Mejia et al., 2005).

A toxina botulínica é uma proteína que possui a capacidade de estimular o desenvolvimento de resposta imune através da formação dos anticorpos neutralizantes (Domingos, 2006). Esses anticorpos promovem o bloqueio dos efeitos terapêuticos da medicação, e por esse motivo, é muito importante evitar a formação desses anticorpos, principalmente quando a toxina é utilizada de forma terapêutica para tratar certos tipos de doenças (Domingos, 2006). O desenvolvimento dos anticorpos que gerarão uma resposta imunológica dependerá da quantidade de proteína existente em cada dose que será administrada na musculatura, por isso, quando pequenas dosagens são aplicadas a chance do paciente desenvolver essa resposta é bem menor (Domingos, 2006).

A toxina botulínica vem sendo comumente utilizada no tratamento de algumas doenças e seu uso vem crescendo cada vez mais como opção de terapia para a paralisia facial, uma vez que trata-se de uma técnica minimamente invasiva que possibilita devolver a simetria facial perdida (Cooper, Lui, \& Nduka, 2017). As complicações no tratamento com a TxB raramente acontecem e dependerão da dose que foi administrada sobre a musculatura, e mesmo quando ocorrem elas são de baixa intensidade e reversíveis, ou seja, melhoram quando o efeito da toxina se findar (Scott, 1980).

\subsection{Aplicabilidades clínicas}

O número de estudos e pesquisas realizadas sobre a toxina botulínica e sua aplicabilidade clínica vem aumentando, o que pode ser explicado pelo grande interesse no campo orofacial em que a TxB pode ser utilizada de forma ampla para auxiliar em vários tipos de doenças que estão relacionadas com a disfunção anatômica e hiperfunção da musculatura (Serrera-Figallo et al., 2020).

O bruxismo, definido como uma atividade mastigatória que ocorre repetidamente é caracterizado pelo hábito de apertar ou ranger os dentes que pode vir acompanhado com a prática de empurrar a mandíbula, e é uma das patologias que podem ser minimizadas com a TxB (Lobbezoo et al., 2018). Com a aplicação da neurotoxina de forma terapêutica nesses casos, se tem resultado satisfatório através da diminuição da atividade dos músculos envolvidos nesse distúrbio, sendo eles o masseter, pterigóideo temporal e lateral (Serrera-Figallo et al., 2020). Além disso, a toxina botulínica também possui propriedade analgésica, o que é de grande importância para tratar e diminuir a dor provocada pelo bruxismo (Serrera-Figallo et al., 2020).

Em casos em que ocorre o deslocamento da ATM, caracterizado pelo completo afastamento das superfícies da articulação temporomandibular, normalmente acompanhado por uma série de sintomas, entre eles a dor, quando o processo se torna crônico a aplicação da TxB pode ser uma das opções de tratamento passadas ao paciente (Oztel, Bilski, \& Bilski, 2017; Prechel, Ottl, Ahlers, \& Neff, 2018). Sendo essa uma alternativa segura e conservadora para tratar o deslocamento da ATM (Oztel et al., 2017).

A TxB pode ser utilizada como forma de tratamento para distonia orofacial, que trata-se de um distúrbio em que ocorre contração da musculatura de forma involuntária e de maneira excessiva, que provoca um caráter fora da normalidade que pode vir acompanhado com movimentos repetitivos (Jinnah \& Factor, 2015).

As queixas de pacientes que apresentam dor orofacial são mais comuns do que se imagina e vem aumentado, principalmente com a pandemia, sendo uma alteração que pode ser tratada com a utilização da TxB (Abboud, Hassin-Baer, Joachim, Givol, \& Yahalom, 2017). As disfunções Temporomandibulares (DTM) estão associadas às dores orofaciais também, mesmo que ainda os resultados não estão bem definidos do uso da toxina para o tratamento destas disfunções, em decorrência dos diferentes efeitos obtidos em testes clínicos (Abboud et al., 2017). As DTMs não apresentam tratamento padrão, pois a 
etiologia é multifatorial e está diretamente relacionada a fatores psicossomáticos, mas quando o paciente não obtém resposta com a realização de tratamentos conservadores, para a sintomatologia dolorosa, uma das opções é a utilização da neurotoxina (Thomas \& Aronovich, 2017). Geralmente as dores orofaciais são consequência das disfunções Temporomandibulares, bruxismos, briquismo, e hábitos parafuncionais relacionados ao sistema estomatognático (Abboud et al., 2017).

Os cistos salivares são lesões que ocorrem nas glândulas produtoras de saliva por questões iatrogênicas, póstraumática e também sem causa definida, levando como consequência ao paciente o surgimento de fístulas ou sialocele, que trata-se do acúmulo de extravasamento de saliva em uma cavidade subcutânea (Serrera-Figallo et al., 2020; Steffen, Hasselbacher, Heinrichs, \& Wollenberg, 2014). Como forma auxiliar no tratamento dessas lesões, a TxB pode ser administrada como uma das opções terapêuticas eficazes dentre diversas outras possibilidades de tratamento, visto que ela tem a capacidade de reduzir temporariamente o fluxo salivar (Steffen et al., 2014).

A sialorréia, ou seja, o fluxo excessivo de salivação é um dos sintomas que acometem muitos indivíduos com doenças neurológicas (Barbero et al., 2016). Complicações como infecções na cavidade oral, pneumonia por aspiração, maceração da pele e o aparecimento de cárie na superfície dos dentes podem surgir em pacientes que apresentam esse distúrbio, o que acaba interferindo de forma negativa na qualidade de vida desses indivíduos (Barbero et al., 2016). Com o intuito de bloquear a liberação de acetilcolina localmente e a atividade das glândulas salivares, passou-se a utilizar a toxina botulínica como opção de tratamento, sendo ela aplicada nas glândulas salivares maiores (Barbero et al., 2016). Assim a produção salivar é reduzida diminuindo o risco de complicações e melhorando a qualidade de vida dos pacientes (Barbero et al., 2016).

A toxina botulínica também pode ser usada no tratamento da Síndrome de Frey, uma complicação que ocorre após cirurgias realizadas nas glândulas salivares, podendo também ocorrer por trauma, ritidoplastia e cirurgia na região do pescoço (Motz \& Kim, 2016). Mesmo que a injeção da TxB não seja um tratamento definitivo para essa síndrome, ela pode reduzir de forma significativa a gravidade da doença, diminuindo assim a morbidade (Motz \& Kim, 2016).

Os efeitos positivos da aplicação da toxina botulínica em diversos estudos podem mostrar uma forma de tratamento muito eficiente e segura para tratar pacientes que possuem neuralgia do trigêmeo sem causa definida, principalmente quando existe a resistência a medicamentos (Park \& Park, 2017). A neuralgia do nervo trigêmeo influencia diretamente na qualidade de vida do indivíduo, sendo ela uma das síndromes dolorosas mais incapacitantes que ocorrem na face (Park \& Park, 2017).

Outra doença que pode contar com os benefícios produzidos pela toxina botulínica são os espasmos hemifaciais (EHF) (Yuksel et al., 2019). Trata-se de uma doença crônica que atinge a musculatura inervada pelo nervo facial ipsilateral, promovendo contrações involuntárias tônicas e clônicas (Yuksel et al., 2019). A aplicação da TxB é a forma de tratamento mais eficaz para realizar o tratamento desse espasmo (Yuksel et al., 2019). Por esse motivo é um dos métodos mais usados no tratamento, já que ela tem a capacidade de controlar os espasmos de forma efetiva, raramente causando complicações (Xiao et al., 2016).

A toxina botulínica também é eficaz e muito utilizada com finalidade estética, podendo ser aplicada para evitar o aparecimento de rugas e para pacientes que possuem sorriso gengival (Dall'Magro et al., 2015). Nos casos de sorriso gengival, para os pacientes que querem melhorar a harmonia do sorriso e não ter a gengiva exposta, a aplicação da TxB pode ser uma das opções de tratamento muito eficiente que diminuirá a hiperatividade dos músculos elevadores do lábio (Dall'Magro et al., 2015). A aplicação da toxina é recomendada quando durante o sorriso mais de $5 \mathrm{~mm}$ de gengiva ficarem expostas, sendo uma alternativa de tratamento menos invasiva que substitui o tratamento cirúrgico em determinados casos (Dall'Magro et al., 2015).

As aplicabilidades clínicas da toxina botulínica são inúmeras, mas a mais conhecida popularmente é a injeção do produto com finalidade estética para fazer o tratamento de rugas (Martins, Silveira, Neto, Martins, \& Pessoa, 2017). Ela é grandemente utilizada por se tratar de um procedimento minimamente invasivo, dispensando tratamentos cirúrgicos em alguns casos, sendo uma possibilidade de procedimento estético que possui efeitos por certo período de tempo, não sendo definitivo 
(Martins et al., 2017). Como vantagem, ela permite controlar melhor os resultados e conseqüências, e quando aplicado por profissional capacitado, minimiza chances de ocorrer efeitos adversos (Martins et al., 2017). A toxina botulínica na maioria das vezes é aplicada na região superior da face, na área das rugas frontais, glabelares e periorbitais, mas também pode ser aplicada em outras áreas, como na pálpebra inferior e no canto do lábio (Martins et al., 2017).

A paralisia facial crônica provoca degeneração dos músculos faciais do lado afetado, o que pode dar ao indivíduo uma aparência mais envelhecida quando comparado com a sua idade real (Choe, Kim, Han, \& Kim, 2017). É uma doença que interfere de forma significativa na qualidade de vida do indivíduo, uma vez que ele tem que lidar com deficiências funcionais e deformidades estéticas, o que acaba interferindo no convívio social e provocando grande desconforto (Choe et al., 2017). A funcionalidade normal da face, no lado não afetado, facilita a percepção dos outros quanto à deficiência, e quanto mais expressivo for a forma de comunicação do indivíduo, com expressões da mímica facial bem definidas, aumenta e agrava a assimetria facial (Choe et al., 2017).

Uma das formas de minimizar a assimetria na paralisia facial de maneira simples e minimamente invasiva é através da aplicação da toxina botulínica, sendo ela altamente eficaz fazendo com que o paciente fique mais confiante para se relacionar em sociedade (Sundaram et al., 2016). Mesmo que seja uma das formas de tratamento comumente utilizadas, não existe um protocolo único de tratamento, mas ela é totalmente eficiente para combater a atividade ou hiperatividade muscular, os espasmos e sincinesias (Sundaram et al., 2016).

\subsection{Paralisia facial}

O sétimo nervo craniano faz o controle de 17 músculos, que são responsáveis pela movimentação facial e por expressar as emoções através da face (Maio \& Soares, 2007). Esse nervo promove a inervação dos músculos envolvidos na mímica facial, pelas contrações que ocorrem de forma voluntária e involuntária das hemifaces e pelo tônus quando em repouso (Maio \& Soares, 2007).

Os danos causados ao nervo facial podem gerar problemas em diferentes níveis, sendo que todos eles interferem de forma significativa na vida do paciente, uma vez que quando lesionado pode gerar tanto distúrbios funcionais como estéticos (Maio \& Soares, 2007).

$\mathrm{O}$ acometimento de qualquer segmento do nervo facial afeta a mímica facial de forma completa ou parcial, podendo estar vinculado a distúrbios na salivação, lacrimejamento e gustação, bem como hiperacusia e hipoestesia do canal auditivo externo (Garanhani, Cardoso, Capelli, \& Ribeiro, 2007).

As causas da ocorrência da paralisia facial são diversas, e elas podem ocorrer por traumas, infecções, processos neoplásicos, por questões congênitas, neurológicas, metabólicas, tóxicas, iatrogênicas e também ocorrem sem causa definida (Salles, 2006). Dentre tantas etiologias da ocorrência da paralisia facial, as causas mais comuns são o AVC (acidente vascular cerebral), as lesões causadas no nervo durante cirurgias ou por algum tipo de trauma, e a mais frequente de todas elas é a idiopática (sem causa definida) (Bento \& Brito, 2004). A etiologia menos comum de causar a paralisia facial são as anomalias que ocorrem durante o desenvolvimento (Bento \& Brito, 2004). Várias doenças e anormalidades apresentam a paralisia facial como sua manifestação final (Salles, 2006).

A paralisia facial também pode estar associada a algumas doenças e condições que o paciente apresente, como diabetes, pressão alta, gravidez, viroses e puerpério (Garanhani et al., 2007).

As etiologias são várias, mas independente de qual delas gerou a paralisia o paciente irá apresentar diminuição ou ausência da mímica facial, mudanças na fala, prejuízos na salivação, além de que o paciente perde a capacidade de assobiar e inflar as bochechas (Salles, 2006). 
As características são comuns nos pacientes que apresentam paralisia na face, e por isso as manifestações são semelhantes entre todos eles (Bento \& Brito, 2004; Maio \& Soares, 2007). Como consequência da paralisia facial, o lado afetado apresenta pequena quantidade de rugas, o sulco nasolabial tem pouca evidência, e ocorre a queda da comissura labial e do supercílio (Bento \& Brito, 2004; Maio \& Soares, 2007). A falta de tônus do lado afetado pela paralisia faz com que o lado não paralisado tenha uma reação hipercinética, o que faz com que surjam desvios faciais que podem ser vistos quando o indivíduo encontra-se em repouso e com mais evidência quando sorri (Bento \& Brito, 2004; Maio \& Soares, 2007).

O nervo facial pode se recuperar em diferentes níveis de forma a restabelecer sua função, mas isso dependerá do que causou a paralisia, do tipo de lesão, da idade do paciente, da nutrição do nervo, de qual foi o comprometimento neuromuscular e a forma de tratamento definida (Garanhani et al., 2007). O nervo facial lesionado pode demorar semanas ou anos para se recuperar, podendo chegar a até 4 anos (Garanhani et al., 2007).

As sequelas provocadas pela paralisia facial interferem diretamente na qualidade de vida do indivíduo e podem causar problemas psicológicos, uma vez que traz tanto prejuízos funcionais como estéticos (Salles, 2006). Mesmo após conseguir uma melhora funcional, grande parte dos pacientes buscam uma melhoria estética, uma vez que a aparência influencia de forma significativa na auto-estima (Salles, 2006). Por isso tem sido muito considerado e levado em conta pelos profissionais que fazem o tratamento desses pacientes, de forma a oferecer condições que melhorem a qualidade de vida e o convívio social (Salles, 2006).

As sincinesias são consideradas as sequelas mais comuns de ocorrer nesses pacientes, e caracteriza-se por movimentos involuntários que ocorrem associados a um movimento da forma voluntária de grupos musculares que são independentes (Freitas \& Gómez, 2008). Pode-se mencionar dentre os tipos de sincinesia, o fechamento dos olhos de forma involuntária durante o sorriso, bem como a sincinesia oral que provoca a movimentação da região da boca quando são realizados movimentos voluntários da testa ou olhos (Freitas \& Gómez, 2008).

Perceber suas próprias sincinesias é um papel difícil para muitos pacientes e quando ela é percebida eles acabam criando formas de evitar as distorções causadas, o que gera uma face com aparência congelada (Freitas \& Gómez, 2008). Isso porque esses pacientes começam a se sentir desconfortáveis ao realizar atos absolutamente normais, como piscar, comer, falar e sorrir, afetando drasticamente o bem-estar e qualidade de vida (Freitas \& Gómez, 2008).

Para tratar a paralisia facial não é simples, e a forma de tratamento dependerá do estágio em que a doença se encontra (Garanhani et al., 2007). A terapia envolve uma série de profissionais como médicos, fisioterapeuta e fonoaudiólogo para ajudar o indivíduo a melhorar as alterações geradas pela paralisia (Garanhani et al., 2007).

Os tratamentos que são feitos após a fase aguda são diversos, como por exemplo, a realização de enxerto de nervo e transferências musculares (Bernardes, Gomez, Pirana, \& Bento, 2004; Maio \& Soares, 2007). São várias as formas de tratar, mesmo que elas não consigam devolver e nem dar o equilíbrio facial almejado ao paciente (Bernardes et al., 2004; Maio \& Soares, 2007). Para melhorar o resultado estético é feita a associação de várias técnicas, como as ritidoplastias, blefaroplastias e a correção de lagoftalmia (Bernardes et al., 2004; Maio \& Soares, 2007).

Em decorrência da hipercinesia dos músculos do lado não paralisado, mesmo com a realização de tratamentos clínicos e cirúrgicos os pacientes apresentam assimetria da face tanto com a musculatura em repouso como em movimento (Maio \& Soares, 2007). Para controlar a hipercinesia e melhorar a assimetria da face de pacientes com paralisia facial, existe além das terapias cirúrgicas, a possibilidade de aplicação da toxina botulínica (Maio \& Soares, 2007). Com a utilização da TxB o paciente apresentará uma redução da assimetria facial (Maio \& Soares, 2007). 


\section{Discussão}

Vem crescendo grandemente a quantidade de estudos realizados a respeito do uso da toxina botulínica, e isso se dá pelo interesse que vem surgido e aumentando cada vez mais no campo orofacial (Serrera-Figallo et al., 2020). O uso da neurotoxina para melhorar a assimetria da face em pacientes com paralisia facial é um procedimento menos invasivo, muito eficiente e que apresenta alto índice de satisfação para os pacientes (Serrera-Figallo et al., 2020). Por isso, vem sendo comumente utilizado, mesmo que ainda não exista um protocolo único de aplicação (Serrera-Figallo et al., 2020). Isso mostra a necessidade da realização de mais estudos científicos com o objetivo de unificar a forma de aplicação, definindo assim um protocolo que poderá ser seguido pelos profissionais de maneira segura e visando minimizar erros.

A reabilitação dos pacientes que possuem a paralisia facial tem o intuito de melhorar a assimetria quando o indivíduo se encontra em repouso e também durante a mímica facial tanto voluntária como involuntária (Bento \& Brito, 2004; Maio \& Soares, 2007; Ueda, Harii, Asato, Yoshimura, \& Yamada, 1999). Dentre as formas de recuperar a simetria da face estão os métodos cirúrgicos como forma de tratamento, em que se pode citar os enxertos, excisões, transposição muscular, transplantes de nervos e músculos, entre outros (Bento \& Brito, 2004; Maio \& Soares, 2007; Ueda et al., 1999).

A grande desvantagem dos tratamentos cirúrgicos são que eles deixam sequelas estéticas no paciente, que vão desde cicatrizes, correções incompletas e recorrências, até parestesias (Maio \& Soares, 2007). Além disso, podem aparecer sincinesias que dificultam ainda mais o tratamento do paciente com paralisia facial (Maio \& Soares, 2007). Na maioria das vezes são realizados vários procedimentos, e os melhores resultados são alcançados quando as técnicas são associadas (Choo, Carter, \& Seiff, 2000; Shumrick \& Pensak, 2000).

Além dos procedimentos cirúrgicos, outra forma de reabilitar esses pacientes é através da aplicação da toxina botulínica, que tem a capacidade de provocar inibição química da musculatura de forma reversível (Finn, 2004; Maio \& Soares, 2007). Isso permite que ela também seja utilizada como forma de teste terapêutico para que depois seja alterada a função muscular de forma definitiva (Finn, 2004; Maio \& Soares, 2007). A TxB é uma das possibilidades de melhorar a assimetria, sendo o tratamento escolhido para os efeitos motores e autonômicos advindos da regeneração nervosa aberrante (Maio \& Soares, 2007).

Nos estudos realizados sobre a aplicação da toxina botulínica em pacientes com paralisia facial fica claro que ela possui um índice alto de sucesso para promover o alívio dos sintomas de forma temporária, independente da causa da paralisia (Neuenschwander, Pribitkin, \& Sataloff, 2000). O grande debate sobre esse assunto é que alguns autores preferem os métodos mais invasivos uma vez que os resultados obtidos com as técnicas cirúrgicas são definitivos, e a reabilitação com a TxB nesses pacientes possuem apenas resultados temporários (Neuenschwander et al., 2000). Isso faz com que tenha a necessidade de várias aplicações da droga com o passar do tempo, visto que ela possui um tempo de duração dos seus efeitos (Maio \& Soares, 2007). Entretanto, consideramos que nenhuma forma de terapia pode ser excluída, podendo também ser utilizada em associação com outros procedimentos, podendo assim funcionar como uma terapia complementar para esses pacientes.

A duração dos efeitos da toxina botulínica também é um assunto contraditório entre os autores (Maio \& Soares, 2007). Em um estudo realizado com 18 pacientes com paralisia facial e hipercinesia contralateral na região perioral a duração dos efeitos da droga foi de aproximadamente 6 meses, em que foi realizado o bloqueio da musculatura contralateral em 8 sítios (Maio \& Soares, 2007). Mas na literatura existem diversos estudos em que o tempo de duração dos efeitos da neurotoxina são diferentes, e menores. Por isso pode-se dizer que o tempo de ação da TxB não é definido, podendo variar conforme as doses e sítios de aplicação.

Dentre os diversos estudos que falam sobre a eficácia da toxina botulínica tipo A na reabilitação de pacientes com paralisia facial, fica difícil realizar uma comparação pelo fato das diferentes características de cada um deles (Cunha, Aguirrei, \& Dias, 1998). Isso porque a quantidade, número e localização das injeções de TxB não são padronizadas (Cunha et al., 1998). 
A maneira de avaliar a duração dos efeitos da droga também é diferente entre os estudos, mas grande parte dos autores levam em consideração apenas os relatos passados pelos pacientes, mesmo que seja um dado subjetivo (Cunha et al., 1998).

É visto a escassez de dados que possam definir e padronizar de forma nacional a reabilitação dos pacientes com paralisia facial utilizando a toxina botulínica (Cooper et al., 2017). Novos trabalhos e pesquisas podem ajudar grandemente na definição dos diversos tipos de campos em que a TxB pode ser utilizada de forma terapêutica (Cooper et al., 2017). Portanto, é interessante sugerir novos estudos com o objetivo de padronizar os protocolos de reabilitação.

\section{Considerações Finais}

Devido aos benefícios da toxina botulínica na reabilitação de pacientes com paralisia facial, ela vem sendo muito utilizada e os estudos a respeito do produto estão aumentando cada vez mais. Portanto, pode-se concluir que a TxB é uma das opções terapêuticas para melhorar a assimetria facial nesses pacientes, mesmo que ainda não exista um protocolo único de utilização, o que mostra a necessidade de pesquisas voltadas para essa área.

Entretanto, a realização de novas pesquisas sobre o assunto é de grande importância e fundamental para que se estabeleça um protocolo que poderá ser seguido pelos profissionais da área para reabilitar os pacientes com paralisia facial através da utilização da toxina botulínica. Através desses trabalhos poderá ser padronizada a utilização da TxB de forma terapêutica, definindo assim as doses, número, local de aplicação e duração dos efeitos do produto.

\section{Referências}

Abboud, W. A., Hassin-Baer, S., Joachim, M., Givol, N., \& Yahalom, R. (2017). Localized myofascial pain responds better than referring myofascial pain to botulinum toxin injections. Int J Oral Maxillofac Surg, 46(11), 1417-1423.

Barbero, P., Busso, M., Artusi, C. A., De Mercanti, S., Tinivella, M., Veltri, A. et al. (2016). Ultrasound-guided Botulinum Toxin-A Injections: a method of treating sialorrhea. J Vis Exp, 9(117), 54606.

Bento, R. F., Brito, R. V. (2004). Gunshot wounds to the facial nerve. Otol Neurotol, 25(6), 1009-1013.

Bernardes, D. F. F., Gomez, M. V. S. G., Pirana, S., \& Bento, R. F. (2004). Functional profile in patients with facial paralysis treated in a myofunctional approach. Pro Fono, 16(2), 151-158.

Brennan, A., \& Hickey, M. (2019). Botulinum toxin in women's health: an update. Maturitas, 119(1), 21-24.

Carruthers, A. (2003). History of the clinical use of botulinum toxin A and B. Clin Dermatol, 21(6), 469-472.

Choe, W. J., Kim, H. D., Han, B. H., \& Kim, J. (2017). Thread lifting: a minimally invasive surgical technique for long-standing facial paralysis. HNO, $65(11), 910-915$.

Choo, P. H., Carter, S. R., \& Seiff, S. R. (2000). Upper eyelid gold weight implantation in the Asian patient with facial paralysis. Plast Reconstr Surg, 105(3), 855-859.

Colhado, O. C. G., Boeing, M., \& Ortega, L. B. (2009). Toxina botulínica no tratamento da dor. Rev Bras Anestesiol, 59(3), 366-381.

Cooper, L., Lui, M., \& Nduka, C. (2017). Botulinum toxin treatment for facial palsy: a systematic review. J Plast Reconstr Aesthet Surg, 70(6), 833-841.

Cunha, M. C., Aguirrei, O. P., \& Dias, C. R. S. (1998). Tratamento do espasmo facial unilateral com toxina botulínica tipo A. Arq Bras Oftal, 61(1), 54-60.

Dall'Magro, A. K., Santos, R., Dall’Magro, E., Fior, B., Matiello, C. N., \& Carli, J. P. (2015). Aplicações da toxina botulínica em odontologia. Rev Salusvita, 34(2), 371-382.

Domingos, M. M. (2006). O uso da toxina botulínica em doentes com hipercinesia muscular facial contralateral à paralisia facial. Tese de Doutorado em Ciência, Universidade de São Paulo, São Paulo.

Dressler, D., Saberi, F. A., \& Barbosa, E. R. (2005). Botulinum toxin: mechanisms of action. Arq Neuro psiquiatr, 63(1), $180-185$.

Finn, J. C. (2004). Botulinum toxin type A: fine-tuning treatment of facial nerve injury. J Drugs Dermatol, 3(2), $133-137$.

Freitas, K. C. S., \& Gómez, M. V. G. (2008). Grau de percepção e incômodo quanto à condição facial em indivíduos com paralisia facial periférica na fase de seqüelas. Rev Soc Bras Fonoaudiol, 13(2), 113-118.

Freund, B., \& Schwartz, M. (2003). Temporal relationship of muscle weakness and pain reduction in subjects treated with botulinum toxin A. J Pain, 4(3), $159-165$. 
Garanhani, M. R., Cardoso, J. R., Capelli, A. M. G., \& Ribeiro, M. C. (2007). Fisioterapia na paralisia facial periférica: estudo retrospectivo. Rev Bras Otorrinolaringol, 73(1), 112-115.

Jinnah, H. A., \& Factor, S. A. (2015). Diagnosis and treatment of dystonia. Neurol Clin, 33(1), 77-100.

Lobbezoo, F., Ahlberg, J., Raphael, K. G., Wetselaar, P., Glaros, A. G., Kato, T. et al. (2018). Consenso internacional sobre a avaliação do bruxismo: relatório de um trabalho em andamento. J Oral Rehabil, 45(11), 837-844.

Maio, M., \& Soares, M. F. D. (2007). Toxina Botulínica em Paralisia Facial: um tratamento miminamente invasivo para redução da hipercinesia muscular da região perioral contralateral. Arq Int Otorrinolaringol, 11(1), 28-35.

Marciano, A., Aguiar, U., Vieira, P. G. M., \& Magalhães, S. R. (2014). Toxina botulínica e sua aplicação na odontologia. Rev Iniciaç Cient Universidade Vale Rio Verde, 4(1), 65-75.

Martins, R. R., Silveira, A. M. M., Neto, J. S. R., Martins, J. C. G., \& Pessoa, C. V. (2017). Toxina botulínica tipo A no tratamento de rugas: uma revisão de literatura. Mostr Cient Farm, 3(1). http://publicacoesacademicas.unicatolicaquixada.edu.br/index.php/mostracientificafarmacia/article/view/1271/1038

Mejia, N. I., Vuong, K. D., \& Jankovic, J. (2005). Long-term botulinum toxin efficacy, safety and immunogenicity. Mov Disord, 20(5), 592-597.

Motz, K. M., \& Kim, Y. J. (2016). Auriculotemporal Syndrome (Frey Syndrome). Otolaryngol Clin North Am, 49(2), 501-509.

Neuenschwander, M. C., Pribitkin, E. A., \& Sataloff, R. T. (2000). Botulinum toxin in otolaryngology: a review of its actions and opportunities for use. Ear Nose Throat J, 79(10), 788-789.

Oztel, M., Bilski, W. M., \& Bilski, A. (2017). Botulinum toxin used to treat recurrent dislocation of the temporomandibular joint in a patient with osteoporosis. Br J Oral Maxillofac Surg, 55(1), e1-e2.

Park, J., \& Park, H. J. (2017). Botulinum Toxin for the Treatment of Neuropathic Pain. Toxins, 9(9), 260-275.

Pereira, A., Shitsuka, D. M., Parreira, F. J., \& Shitsuka, R. (2018). Metodologia da Pesquisa Científica. UFSM https://repositorio.ufsm.br/bitstream/handle/1/15824/Lic_Computacao_Metodologia-Pesquisa-Cientifica.pdf?sequence=1

Prechel, U., Ottl, P., Ahlers, O. M., \& Neff, A. (2018). The Treatment of Temporomandibular Joint Dislocation. Dtsch Arztebl Int, 115(5), 59-64.

Salles, A. G. (2006). Avaliação do efeito da toxina botulínica no lado são em pacientes com paralisia facial de longa duração. Tese de Doutorado em Ciência, Universidade de São Paulo, São Paulo.

Scott, A. B. (1980). Botulinum toxin injection into extraocular muscles as an alternative to strabismus surgery. Ophthalmology, 87(10), 1044-1049.

Serrera-Figallo, M. A., Ruiz-de-León-Hernández, G., Torres-Lagares, D., Castro-Araya, A., Torres-Ferrerosa, O., Hernández-Pacheco, E. et al. (2020). Use of Botulinum Toxin in Orofacial Clinical Practice. Toxins, 12(2), 1-16.

Shumrick, K. A., \& Pensak, M. L. (2000). Early perioperative use of polytef suspension for the management of facial paralysis after extirpative skull base surgery. Arch Facial Plast Surg, 2(4), 243-248.

Steffen, A., Hasselbacher, K., Heinrichs, S., \& Wollenberg, B. (2014). Botulinum toxin for salivary disorders in the treatment of head and neck cancer. Anticancer Res, 34(11), 6627-6632.

Sundaram, H., Signorini, M., Liew, S., Almeida, A. R. T., Wu, Y., Braz, A. V. et al. (2016). Consenso Global de Estética: toxina botulínica tipo A - revisão baseada em evidências, conceitos emergentes e recomendações de consenso para uso estético, incluindo atualizações sobre complicações. Plast Reconstr Surg, $137(3), 518 \mathrm{e}-529 \mathrm{e}$.

Thomas, N. J., \& Aronovich, S. (2017). Does Adjunctive Botulinum Toxin A Reduce Pain Scores When Combined With Temporomandibular Joint Arthroscopy for the Treatment of Concomitant Temporomandibular Joint Arthralgia and Myofascial Pain?. J Oral Maxillofac Surg, 75(12), $2521-2528$.

Ueda, K., Harii, K., Asato, H., Yoshimura, K., \& Yamada, A. (1999). Evaluation of muscle graft using facial nerve on the affected side as a motor source in the treatment of facial paralysis. Scand J Plast Reconstr Surg Hand Surg, 33(1), 47-57.

Unno, E. K., Sakata, R. K., \& Issy, A. M. (2005). Estudo comparativo entre toxina botulínica e bupivacaína para infiltração de pontos-gatilho em síndrome dolorosa miofascial crônica. Rev Bras Anestesiol, 55(2), 250-255.

Xiao, L., Pan, Y., Zhang, X., Hu, Y., Cai, L., Nie, Z. et al. (2016). Facial asymmetry in patients with hemifacial spasm before and after botulinum toxin A treatment. Neurol Sci, 37(11), 1807-1813.

Yuksel, B., Genc, F., Yaman, A., Goksu, E. O., Ak, P. D., \& Gomceli, Y. B. (2019). Evaluation of stigmatization in hemifacial spasm and quality of life before and after botulinum toxin treatment. Acta Neurologica Belgica, 119(1), 55-60. 